\title{
Aceptación y confianza de Bitcoin en México: una investigación empírica $^{1}$
}

\section{Acceptance and Trust of Bitcoin in México: An empirical research}

\author{
C.R. López y M. Camberos \\ Recibido: junio 1 de 2020 - Aceptado: noviembre 16 de 2020.
}

\begin{abstract}
Resumen- El Bitcoin forma parte de las llamadas criptomonedas y puede definirse como un sistema de pagos electrónico público y descentralizado universal, basado en la tecnología de Blockchain. El problema que enfrenta es la confianza en su uso; por lo tanto, el objetivo de la presente investigación es analizar los factores que influyen la adopción y uso de Bitcoin en México. Para ello, se utiliza el modelo UTAUT2 integrado con la variable de confianza. El modelo fue probado empíricamente mediante la aplicación de una encuesta de 106 cuestionarios, bajo el método de mínimos cuadrados parciales, con modelos de ecuaciones estructurales (PLS-SEM). Los principales resultados indican que la expectativa de rendimiento, la motivación hedonista, el hábito y su descentralización, son los principales factores que influyen en la confianza para la adopción y uso de Bitcoin. Los resultados ilustran a las empresas y cualquier interesado sobre las ventajas del uso de Bitcoin.
\end{abstract}

Palabras clave- Aceptación, Bitcoin, Criptomonedas, Confianza y UTAUT2

Abstract-Bitcoin is part of the so-called crypto-currencies and can be defined as a universal decentralized public electronic payment system based on Blockchain technology. The problem it faces is trust in its use; therefore, the objective of this research is to analyze the factors that influence the adoption and use of Bitcoin in Mexico. For this purpose, the UTAUT2 model

\footnotetext{
${ }^{1}$ Producto derivado del proyecto de investigación "Aceptación del Bitcoin: una cuestión de confianza". Presentado por el Grupo de Investigación del Centro de Investigación en Alimentación y Desarrollo (CIAD) en Hermosillo, Sonora.

C.R. López, Centro de Investigación en Alimentación y Desarrollo (CIAD), Hermosillo, Sonora, email: carlos.lopezdr18@estudiantes.ciad.mx.

M. Camberos, Centro de Investigación en Alimentación y Desarrollo (CIAD), Hermosillo, Sonora, email: mcamberos@ ciad.mx.
}

Como citar este artículo: López, C. R. y Camberos, M. Aceptación y confianza de Bitcoin en México: una investigación empírica, Entre Ciencia e Ingeniería, vol. 14 , no. 28 , pp. 16-25, julio-diciembre, 2020. DOI: https://doi.org/10.31908/19098367.2011.

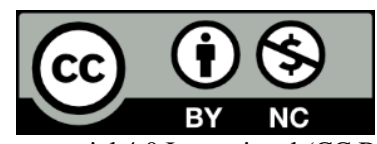

Attribution-NonCommercial 4.0 Intenational (CC By-NC 4.0) integrated with the trust variable, is used. The model was empirically tested by applying a survey of 106 questionnaires, under the partial least square's method, with structural equation models (PLS-SEM). The main results indicate that performance expectation, hedonistic motivation, habit, and its decentralization; are the main factors that influence confidence for the adoption and use of Bitcoin. The results illustrate to companies and any interested party the advantages of using Bitcoin.

Keywords-Acceptance, Bitcoin, Cryptocurrencies, Trust, UTAUT2.

\section{INTRODUCCIÓN}

$\mathrm{N}$ el mundo de la economía digital, la aparición del Bitcoin y las criptomonedas de la mano de Satoshi Nakamoto en 2008 demuestran ser una de las tecnologías financieras más innovadoras y novedosas del mercado, presentándose E como una competencia directa del dinero fiduciario [1]. El Bitcoin es un sistema electrónico de pagos anónimo [2] que se basa en Blockchain, esta tecnología ha permitido un cambio radical en las formas en la que se prestan los servicios financieros ya que la disponibilidad de esta innovación tecnológica ha permitido el auge de las criptomonedas, actualmente existen más de 5,000 de ellas en los mercados FinTech (financieras tecnológicas) [3], ofreciendo una variedad de servicios, desde contratos inteligentes hasta ofertas iniciales de monedas (OIM) o simplemente son usadas como medios de pago que la convierten en un nuevo medio de inversión en capital de riesgo [4].

El valor total del mercado de las criptomonedas es de 250 mil millones de dólares y es dominado en un $65.5 \%$ por Bitcoin, convirtiéndola en la criptomoneda más importante [3]. A excepción de Ecuador donde el uso de Bitcoin es ilegal, la mayoría de los países en América Latina cuentan con leyes Fintech que regulan su uso, tal es el caso de México, Brasil y Chile [5]. La naturaleza anónima de Bitcoin dificulta localizar a los usuarios de Bitcoin, en un estudio [6], se menciona que existen al menos 35 millones de usuarios confirmados aunque esta cifra puede elevarse fácilmente a 126 millones [6].

El Bitcoin es lanzado como una evolución de los servicios 
financieros, el éxito de esta tecnología deriva de una gran aceptación por parte de las personas convirtiéndose en el factor más crítico para impulsar a la criptomoneda como mecanismo de pago generalizado [1], [7]. La literatura existente demuestra que la aceptación de las criptomonedas ha sido abordada en diferentes regiones, sin embargo, la evidencia empírica aun es limitada y se necesita ampliar para entender cuáles son los principales factores que deriven en su aceptación, por lo que la brecha en las investigaciones relacionadas con la aceptación del Bitcoin se puede resumir en la necesidad de proponer un modelo conceptual que refleje los principales factores de la adopción de Bitcoin [1], [8]-[16] . Además, es necesario aplicar modelos estadísticos complejos con técnicas de segunda generación como el modelo de ecuaciones estructurales (SEM) [17], [18].

Por lo tanto, el objetivo de este trabajo es proponer un modelo de investigación basado en la teoría unificada de la aceptación y uso de la tecnología (UTAUT2) que examine empíricamente los factores más importantes que derivan en la aceptación de Bitcoin con la finalidad de conocer que necesita este para ser aceptado a pesar de carecer de respaldo institucional. También en este estudio se introduce la confianza al modelo UTAUT2 para profundizar el alcance de los predictores de la aceptación [17]. Adicionalmente, cabe aclarar, que no se han realizado investigaciones previas con respecto a la aplicación del modelo UTAUT2 para estudiar la adopción y el uso de Bitcoin en México; por lo tanto, esta investigación será pionera. Para ello, se propone el desarrollo en las siguientes etapas. En la primera se describe el concepto de Bitcoin, las teorías actuales que explican la aceptación y uso de la tecnología, así como una revisión de investigaciones previas sobre este tema. Después se presenta el modelo conceptual, la metodología, luego los resultados y una discusión que incluye las contribuciones teóricas y sus implicaciones y finalmente las conclusiones.

\section{MARCO TEÓRICO}

\section{A. El Bitcoin}

El Bitcoin ha surgido como una nueva alternativa a los medios de pago que representa múltiples beneficios para los usuarios como anonimidad y bajas tarifas de transacción [1]. Es un sistema de pagos público y descentralizado que se basa en la tecnología de Blockchain, consiste en un registro de todas las transacciones que se llevan a cabo a través de él, es un sistema criptográfico ordenado en bloques en el que cada bloque contiene el registro de transacciones que son llevadas a cabo en un lapso de tiempo, cada bloque confirmado contiene las marcas temporales de cada transacción haciéndolas únicas que evitan el doble gasto, una vez verificado por toda la red el bloque se añade a la cadena de bloques, la cadena de bloques es la secuencia de todos los bloques ya confirmados que sirven de referencia válida para las cadenas posteriores y evitan transferencias duplicadas [19]. En otras palabras, es un libro contable abierto en el que se van registrando todas las operaciones y en el que todos pueden participar [4].

Para poder comprender con mayor exactitud el sistema de pagos del Bitcoin es necesario conocer algunos conceptos básicos que se describen a continuación: 1) Las transacciones, son los registros de todas las transferencias que se hacen entre los usuarios, llevan la marca temporal que evita que un solo Bitcoin sea gastado dos veces [19]; 2) las direcciones de bitcoin, son claves se asocian a una dirección específica de la cadena de bloques y se gestionan a través de monederos virtuales o billeteras; 3) Los monederos o billeteras, consisten en carteras digitales que sirven para almacenar e enviar los Bitcoins [20] y; 4) la minería en Bitcoin exige que todos los procedimientos del sistema de pagos, que necesitan llevar a cabo procesos criptográficos, sean realizados por usuarios voluntarios que aportan capacidad de proceso a través de sus computadoras, ellos confirman las transacciones y procesan los nuevos bloques que se añaden a la cadena, todas las transacciones y nuevos bloques generan comisiones que son repartidas equitativamente entre los participantes [21].

\section{B. Los modelos de adopción de tecnologías}

La teoría unificada de la aceptación y uso de la tecnología consiste en un modelo que unifica ocho teorías intrínsecamente relacionadas, en la referencia [22] se realiza un estudio en un contexto organizacional en el que analiza los conceptos fundamentales de cada teoría y los compara empíricamente con la finalidad de conocer cuáles son los conceptos más significantes y determinantes de la aceptación de los usuarios. Este modelo postula cuatro constructos como principales determinantes de la intención y del comportamiento de uso, consisten en la expectativa de rendimiento, la expectativa de esfuerzo, la influencia social y las condiciones de facilitación [22], este modelo ha sido ampliamente aplicado en el área de banca móvil y de banca por internet con resultados consistentes y robustos [23]-[26].

En suma este modelo trata de predecir la intención del usuario y el comportamiento subsecuente sobre la tecnología, determina que el efecto positivo de la expectativa de rendimiento, la expectativa de esfuerzo, las condiciones de facilidad y de la influencia social sobre la intención de uso derivan en el uso continuo de la tecnología y por lo tanto su aceptación [27], este modelo no solo ayuda a analizar los factores que influencian la aceptación sino también a entenderlos [26]. El modelo UTAUT precisa los factores más críticos relacionados con la predicción de la intención de uso, como también, en contextos más organizacionales; por ello, en la referencia [28] se hace un análisis con el propósito de hacer una mejora sustancial al modelo UTAUT y con la finalidad de adaptar el modelo al contexto del consumidor incorpora tres nuevos constructos a la teoría introduciendo nuevas relaciones: 1) motivación hedonista, 2)valor del precio y 3 ) habito, sumándole a estos diferencias individuales como efectos moderadores en la intención de uso que son la edad, el género y la experiencia, En suma las variables del modelo UTAUT2 contribuyen a la intención de uso del individuo hacia la adopción de la tecnología, dicho de otra forma la intención de adoptar la tecnología en cuestión llevara finalmente al comportamiento de uso real [1]. Este modelo ha sido probado extensamente en el área de banca móvil combinándolo exitosamente con otras teorías y añadiendo nuevos constructos que permiten la extensión y mayor conocimiento según el área en cuestión [29]-[31] y en menor medida en el área de criptomonedas con resultados de validez y confianza exitosos [1], [13], [32]. 


\section{Evidencia empírica}

La aceptación en las criptomonedas ha sido analizada de diferentes maneras y a pesar de la aparente madurez que se deriva de la comparación de diferentes estudios, ninguno de ellos hace una exhaustiva aplicación del modelo UTAUT2 (enfocado al consumidor) incluyendo la variable de confianza a través del método SEM. Se encontró que todos los trabajos revisados presentan una adecuada aplicación de las diferentes variables teóricas de los modelos de aceptación, integrándolos con diferentes conceptos o teorías de aceptación como la teoría del comportamiento planeado (TPB), en todos ellos se comprobó exitosamente la confiabilidad y validez de los modelos. El concepto más recurrente con el que se integran los modelos de aceptación, es la confianza, el método más utilizado para analizar los datos es a través del modelo de ecuaciones estructurales (SEM) aunque solo dos trabajos utilizan los mínimos cuadrados ordinarios (PLS) en sus análisis, todos los estudios demuestran resultados significantes que logran explicar el uso y la intención de uso en las criptomonedas destacando que la confianza cuando es analizada es el factor más importante [1], [11], [13], [15], [32]-[35].

Se encontraron tres trabajos que consideran a la confianza como un elemento importante de análisis en la adopción de las criptomonedas, al respecto, en la referencia [14] se realiza un estudio en China sobre la adopción de las criptomonedas utilizando el modelo TAM e integra las variables de confianza y conciencia, siendo la confianza el factor más importante ya que explica el $33 \%$ de la intención de uso. En otro estudio sobre la adopción de criptomonedas en las pequeñas y medianas empresas, Roos (2015) hace una aplicación del modelo UTAUT2 integrando la variable de confianza unidimensional a su modelo, a través del análisis logra obtener resultados favorables en cuanto a la confiabilidad y validez del modelo, este estudio también demuestra que la confianza es el elemento más importante en cuanto a la intención de uso, seguido del hábito. Los únicos datos obtenidos de la presente revisión que son comparables con el presente trabajo de investigación corresponden a Mahomed (2017) quien hace una aplicación del modelo UTAUT2 integrado con la confianza unidimensional, este estudio logra obtener resultados robustos a través de una técnica de primera generación por medio del método de regresión múltiple en los que demuestra que la motivación hedónica es el factor más importante en la intención de uso explicando un $33 \%$ seguido de la confianza que logra explicar el $25 \%$, en total esta aplicación logra explicar un $60 \%$ de la varianza de la intención de uso y un $28 \%$ del uso actual de las criptomonedas aunque este estudio no considera la variable de hábito.

\section{INTEGRACIÓN DEL MODELO CONCEPTUAL}

Se propone un modelo de investigación de adopción del Bitcoin que cubra todos los factores que determinan la intención de uso, se utiliza la base teórica del modelo UTAUT2 integrado con la variable de confianza, este modelo se ilustra en la Fig. 1 [36]. Esta integración se hace asumiendo que la aceptación del Bitcoin es un fenómeno complicado que requiere una perspectiva integradora que proporcione una descripción más completa de los mecanismos causales de las relaciones que no pueden obtenerse de un solo modelo, por lo que se refuerza la importancia y previsibilidad de los resultados [29]. En el presente modelo no se incluyen los moderadores de edad, sexo y experiencia, ya que está fuera del objetivo del presente estudio el análisis comparativo entre grupos de edad y géneros [1], [13], [15].

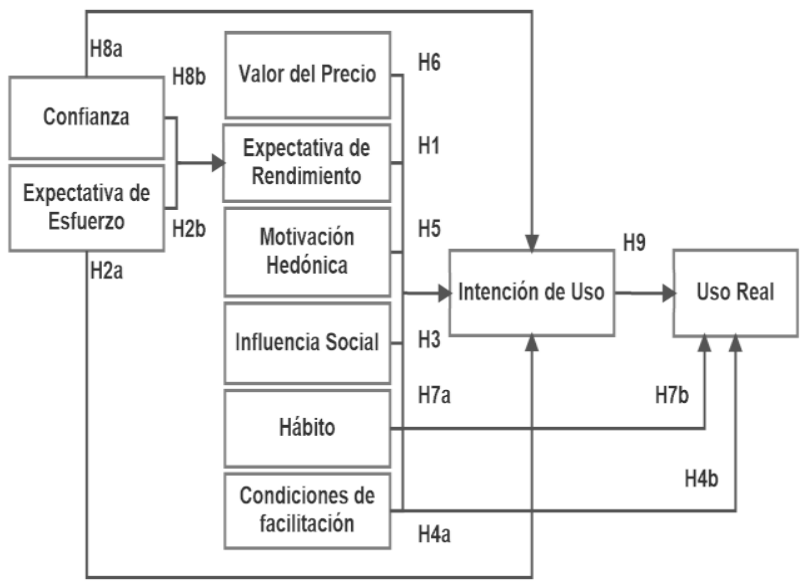

Fig. 1. Modelo integrado (Adaptado de [7]).

La Fig. 1 muestra las relaciones más importantes entre los diferentes conceptos, en las que se puede apreciar la importancia de cada una de ellas, en primer lugar, la confianza que hace posible su uso, concepto final que cierra la Fig. 1. La dirección de las flechas señala la influencia. A partir de ellas se tejen una serie de hipótesis $[\mathrm{H}]$ expuestas a continuación.

La expectativa de rendimiento, se define como el grado en que una persona cree que usando determinada tecnología esta le hará obtener ganancias en el desempeño laboral, [23], [37].

\section{H1. La expectativa de rendimiento (ER) influye positivamente la intención de usar Bitcoin.}

La expectativa de esfuerzo, es definida como el grado de facilidad asociado con el uso de la tecnología [22], [38]. Para usar Bitcoin se requieren conocimientos técnicos y financieros mínimos que faciliten el uso de la tecnología sin mucho esfuerzo [32].

H2a. La expectativa de esfuerzo (EE) tendrá una influencia positiva en la intención de uso de las criptomonedas.

$H 2 b$. La expectativa de esfuerzo (EE) tendrá una influencia positiva en expectativa de rendimiento del Bitcoin.

La influencia social se define como el grado en el que una persona percibe que tan importante es para otros que el utilice o no determinada tecnología o igual que el cumpla con la expectativa de otros [23], [37], [39]. Como un sistema de persona a persona el Bitcoin trabaja sin una autoridad central por lo que el efecto de los factores sociales tienen una influencia significativa en el uso de Bitcoin [1].

\section{H3. La influencia social (IS) influye positivamente en la intención de comportamiento del individuo de adoptar Bitcoin.}

Las condiciones de facilitación, son aquellas definidas como el grado en que una persona cree que existe una estructura organizativa y técnica que soporta a la tecnología [37], [40]. El uso del Bitcoin requiere ciertas habilidades, 
recursos e infraestructura técnica por lo tanto los usuarios podrían estar más motivados para usar Bitcoin si se cuenta con un cierto nivel de servicio y recursos de soporte [30].

H4a. Las condiciones de facilitación (CF) influyen positivamente en la intención de uso de Bitcoin.

$H 4 b$. Las condiciones de facilitación (CF) influyen positivamente en el comportamiento de uso de Bitcoin.

La motivación hedonista, se refiere al grado de diversión o placer derivado del uso de la tecnología, [31], [41]. El Bitcoin es utilizado como un medio de cambio y como un medio de inversión para generar ingreso, cuando se es usada con este fin el individuo puede lograr una sensación de satisfacción cuando se genera el retorno de [1].

H5. La motivación hedonista (MH) tendrá un efecto positivo en la intención de uso del Bitcoin.

El valor del precio se define como la sensación positiva del usuario frente a los beneficios percibidos de las tecnologías frente al costo monetario que implica su uso, [30], [41]. El costo de usar Bitcoin es más bajo comparado con medios de pago similares, por lo que este factor puede tener una influencia positiva en la adopción de Bitcoin.

H6. El valor del precio (VP) tiene una influencia positiva en la intención de uso del Bitcoin.

El hábito es definido como la medida en que las personas tienden a realizar determinados comportamientos debido al aprendizaje que han obtenido con el uso [41]. Todas las personas tienen experiencia en el uso del dinero y existe una gran penetración de la banca móvil, por ello es importante evaluar como el hábito afecta el uso del Bitcoin [1], [27], [30], [39], [42].

H7a. El hábito (HB) tiene una influencia positiva en la intención de uso del Bitcoin.

H7b. El hábito (HB) tiene una influencia positiva en el comportamiento de uso de Bitcoin.

La confianza se puede definir como una combinación de confiabilidad, integridad, benevolencia y capacidad del usuario de una determinada tecnología con la creencia de que se pueden generar intenciones de comportamiento entre los usuarios [30], [36]. La confianza en Bitcoin ayuda al usuario a permitirle descartar subjetivamente situaciones indeseables derivadas de su uso, sobre todo por parte de los prestadores de servicios.

H8a. La confianza (CZ) afecta positivamente la intención de los usuarios de utilizar Bitcoin.

H8b. La confianza afecta positivamente la expectativa de rendimiento de Bitcoin

La intención de uso supone que la adopción real del Bitcoin se puede predecir por la disposición de los usuarios a adoptar esta tecnología [28], [30], [37], [43]..

H9. La intención de uso (IU) afecta positivamente la conducta de uso (UR) del Bitcoin.

\section{Metodología}

\section{A. Medición}

Para probar el modelo se aplicó un cuestionario electrónico elaborado con base en la revisión de literatura. Los ítems y las escalas relacionados con la aceptación fueron adaptados de la referencia [28], los ítems y las escalas relacionados con la confianza fueron adaptados de la referencia [36]. La unidad de análisis se lleva a cabo a través de una escala Likert de 7 puntos en niveles de intervalo que van desde "totalmente en desacuerdo" a "totalmente de acuerdo". La frecuencia de uso se midió a través de una escala de 7 puntos con un rango que va desde "nunca" a "varias veces al día". Se incluyeron preguntas demográficas que se refieren a la edad, género, ingreso y nivel educativo con la finalidad de conocer el contexto.

El cuestionario se elaboró a través de la plataforma de Google Forms, fue creado en idioma español y se validó frente a un panel de expertos en economía y finanzas, posteriormente con la finalidad de hacer una prueba piloto se distribuyó el cuestionario a través de la base de datos de una plataforma llamada Airbit Club que opera en México y maneja diferentes criptomonedas. Se recibieron 21 respuestas que no fueron incluidas en el estudio principal encontrando evidencia preliminar de que las escalas eran validas y confiables.

\section{B. Datos}

Después de la prueba piloto se optó por un enfoque de muestreo no probabilístico por conveniencia, considerándolo como el método más apropiado debido a que no existen datos sobre usuarios de Bitcoin y por ello un universo conocido o aproximado, pero ofrece la ventaja de obtener información confiable sobre el tema de estudio y de elegir encuestados que estén familiarizados con las criptomonedas [17], para lo cual, se decidió distribuir el cuestionario a través de 13 grupos relacionados con criptomonedas de la plataforma Facebook en México, por medio del software AutoNetworks se lograron extraer 4,500 nombres de usuarios de estos grupos a los que se les enviaron mensajes personales con los datos del cuestionario, obteniendo 106 respuestas validadas con instrumentos estadísticos explicados con detalle en la metodología para dar confiabilidad a los resultados.

\section{RESULTAdOS}

El perfil de respuestas se observa en la tabla 1 donde el 95\% corresponde a hombres, la media de edad es de 33 años y el $78 \%$ tiene grado universitario.

El modelo de ecuaciones estructurales (SEM) permite a los investigadores incluir más constructos a sus modelos y dar cuenta de los errores de medición en las variables además permite comprobar teorías, conceptos y verificar las relaciones entre variables a un nivel teórico [17], [44]. Existen dos tipos de modelos SEM, el modelo de ecuaciones estructurales basado en covarianza (CB-SEM) estima los parámetros de manera que minimiza la discrepancia entre matrices de covarianza estimada y de muestra, mientras que el modelo de ecuaciones estructurales de mínimos cuadrados ordinarios (PLS-SEM) maximiza la varianza explicada de las variables al 
estimar las relaciones parciales del modelo en una secuencia de regresión de mínimos cuadrados ordinarios [44].

TABLA I

INFORMACIÓN DEMOGRÁFICA

\begin{tabular}{|l|l|l|}
\hline Información Demográfica & $\#$ & $\%$ \\
\hline Genero & 101 & 95 \\
Masculino & 5 & 5 \\
\hline Edad & & \\
Menores de 20 & 2 & 2 \\
$21-25$ & 28 & 26 \\
$26-35$ & 41 & 39 \\
$36-45$ & 26 & 25 \\
Mayores a 45 & 9 & 8 \\
\hline Educación & & \\
Secundaria & 2 & 2 \\
Preparatoria & 21 & 20 \\
Licenciatura & 67 & 63 \\
Posgrado & 16 & 15 \\
\hline
\end{tabular}

Se evaluó la distribución de la muestra a través de la prueba Kolmogorov-Smirnov (K-S) detectando que los datos no están distribuidos normalmente ya que los niveles de significancia fueron menores a 0.05. Basándonos en esta prueba el modelo de ecuaciones estructurales de mínimos cuadrados ordinarios (PLS-SEM) es el método más apropiado para este estudio añadiendo que la naturaleza anónima del uso de las criptomonedas no permite obtener muestras muy grandes [23]. El modelo de medición reflectiva es el que mejor se adapta a este estudio ya que los indicadores de las variables están altamente correlacionados [45]. Otros de los aspectos que justifican el uso del método PLS-SEM en este estudio se refieren al objetivo de predecir constructos fundamentales (aceptación y confianza) y que el estudio parte de una investigación exploratoria debido al reciente fenómeno de las criptomonedas siendo el software SmartPLS el más adecuado para examinar el modelo [45].

\section{A. Análisis del modelo de medición}

El modelo de medición se evaluó con la finalidad de conocer la confiabilidad y validez de las medidas, utilizando un modelo reflectivo para medir las variables [46]. [46]. Para evaluar el modelo de medición, se calculó la consistencia interna, la confiabilidad del indicador, la validez convergente, el promedio de la varianza extraída (AVE) y la validez discriminante.

La confiabilidad compuesta (CC) mide la consistencia interna cuyos valores deben de ser mayores a 0.70 para ser aceptables [17], [46]. Como se muestra en la Tabla II, todos los valores son mayores a 0.70 . Para evaluar la validez convergente se utiliza la fiabilidad del indicador cuyos valores deben de ser mayores a 0.70 siempre y cuando si al eliminarlo aumenta la confiabilidad compuesta [46]. Como se puede comprobar en la tabla II solo un indicador muestra valores menores a 0.70 , pero su eliminación no causa efecto. También se utiliza el AVE, cuyos valores deben ser mayores a 0.50 indicando que explican más de la mitad de la varianza [46].
La validez discriminante representa la medida en que el constructo mide lo que se pretende, se utiliza método FornellLarcker que establece que cada constructo comparte más varianza con sus indicadores que con cualquier otro constructo, como se observa en la Tabla III el valor de cada variable es mayor que el de su correlación más alta [46].

TABLA II

CONSISTENCIA INTERNA Y VALIDEZ

\begin{tabular}{|c|c|c|c|c|c|}
\hline VARIABLE & ÍTEM & $\begin{array}{c}\text { Alfa de } \\
\text { CRONBACH }\end{array}$ & $\mathrm{CC}$ & AVE & INDICADOR \\
\hline \multirow[t]{3}{*}{$\mathrm{CF}$} & CF1 & 0.716 & 0.841 & 0.646 & 0.88 \\
\hline & $\mathrm{CF} 2$ & & & & 0.91 \\
\hline & CF3 & & & & 0.59 \\
\hline \multirow[t]{4}{*}{$\mathrm{CZ}$} & CZ1 & 0.891 & 0.926 & 0.758 & 0.93 \\
\hline & $\mathrm{CZ2}$ & & & & 0.75 \\
\hline & $\mathrm{CZ3}$ & & & & 0.85 \\
\hline & $\mathrm{CZ} 4$ & & & & 0.94 \\
\hline \multirow[t]{4}{*}{$\mathrm{EE}$} & EE1 & 0.891 & 0.924 & 0.754 & 0.89 \\
\hline & EE2 & & & & 0.87 \\
\hline & EE3 & & & & 0.84 \\
\hline & EE4 & & & & 0.88 \\
\hline \multirow[t]{4}{*}{ ER } & ER1 & 0.924 & 0.946 & 0.814 & 0.92 \\
\hline & ER2 & & & & 0.91 \\
\hline & ER3 & & & & 0.88 \\
\hline & ER4 & & & & 0.9 \\
\hline UR & FREC & & & & 1 \\
\hline \multirow[t]{3}{*}{ HB } & HB1 & 0.781 & 0.872 & 0.694 & 0.86 \\
\hline & HB2 & & & & 0.86 \\
\hline & HB3 & & & & 0.78 \\
\hline \multirow[t]{3}{*}{ IS } & IS1 & 0.866 & 0.917 & 0.786 & 0.83 \\
\hline & IS2 & & & & 0.94 \\
\hline & IS3 & & & & 0.89 \\
\hline \multirow[t]{4}{*}{ IU } & IU1 & 0.903 & 0.933 & 0.776 & 0.89 \\
\hline & IU2 & & & & 0.84 \\
\hline & IU3 & & & & 0.94 \\
\hline & IU4 & & & & 0.85 \\
\hline \multirow[t]{3}{*}{ MH } & MH1 & 0.918 & 0.948 & 0.86 & 0.95 \\
\hline & MH2 & & & & 0.95 \\
\hline & MH3 & & & & 0.88 \\
\hline \multirow[t]{4}{*}{ VP } & VP1 & 0.825 & 0.883 & 0.655 & 0.8 \\
\hline & VP2 & & & & 0.84 \\
\hline & VP3 & & & & 0.76 \\
\hline & VP4 & & & & 0.83 \\
\hline
\end{tabular}

Siguiendo las reglas generales para evaluar el modelo de medición se llega a la conclusión de que los resultados son satisfactorios confirmando la validez y la confiablidad de las 
variables, por lo que pueden ser usadas para probar el modelo estructural.

TABLA III

CRITERIO DE FORNELL-LARCKER

\begin{tabular}{|c|c|c|c|c|c|c|c|c|c|}
\hline VAR & CF & CZ & EE & ER & HB & IU & IS & MH & VP \\
\hline CF & $\mathbf{0 . 8 0}$ & & & & & & & & \\
\hline CZ & 0.58 & $\mathbf{0 . 8 7}$ & & & & & & & \\
\hline EE & 0.71 & 0.61 & $\mathbf{0 . 8 7}$ & & & & & & \\
\hline ER & 0.48 & 0.49 & 0.56 & $\mathbf{0 . 9 0}$ & & & & & \\
\hline HB & 0.56 & 0.55 & 0.52 & 0.61 & $\mathbf{0 . 8 3}$ & & & & \\
\hline IU & 0.65 & 0.81 & 0.64 & 0.64 & 0.68 & $\mathbf{0 . 8 8}$ & & & \\
\hline IS & 0.20 & 0.04 & 0.19 & 0.34 & 0.28 & 0.16 & $\mathbf{0 . 8 9}$ & & \\
\hline MH & 0.55 & 0.65 & 0.60 & 0.59 & 0.58 & 0.74 & 0.13 & $\mathbf{0 . 9 3}$ & \\
\hline VP & 0.50 & 0.54 & 0.59 & 0.51 & 0.50 & 0.59 & 0.21 & 0.51 & $\mathbf{0 . 8 1}$ \\
\hline
\end{tabular}

\section{B. Análisis del modelo estructural}

Una vez que se estableció la confiabilidad y validez, se procede a estudiar el modelo estructural donde se analizan las relaciones de causalidad entre las variables latentes independientes y dependientes [45], a través del coeficiente de determinación $\left(\mathrm{R}^{2}\right)$. La no existencia multicolinealidad se mide con el factor de inflación de la varianza (VIF) que deben oscilar entre 0.20 y 5 [17]. En la Tabla IV se observa que los valores se encuentran en el rango permitido por lo que se puede determinar que no existe multicolinealidad.

La $\mathrm{R}^{2}$ es una medida de la precisión predictiva del modelo, representa el efecto combinado de las variables independientes sobre las dependientes, se determina calculando los cuadrados de las correlaciones entre valores reales y pronosticados de las variables endógenas, este efecto se representa con valores que van de 0 a 1 , siendo uno el que representa la precisión predictiva completa, valores mayores a 0.20 se consideran altos y menores a 0.10 insignificantes [17], [46], [47].

En la Fig. 2, infra, figura compleja de interpretar, se aclara que sintetiza la prueba estadística de las hipótesis, en la que se observa que el modelo explica el $34.7 \%$ de la expectativa de rendimiento confirmando las hipótesis $\mathrm{H} 2 \mathrm{a}$ y $\mathrm{H} 2 \mathrm{~b}$, igualmente explica $79.3 \%$ de la intención de uso siendo la confianza el factor más importante ya que explica un $44.5 \%$, el $30.6 \%$ de la varianza de uso real de Bitcoin, de la que el $65.1 \%$ es explicada por el hábito. La intención de uso tiene una connotación negativa sobre el uso real negando la hipótesis H9 no se confirma. Las hipótesis H1, H2b, H4a, H5, H7a, H7b, H8a, H8b son confirmadas; mientras que las hipótesis H2a, H3, H4b, H6, H9 no son confirmadas. Para terminar este apartado de análisis estadístico de resultados, se utiliza la técnica de remuestreo Bootstrapping para conocer los niveles de significancia de los coeficientes de ruta. Al respecto, en la Tabla V se muestra que la confianza, la expectativa de rendimiento, el hábito y la motivación hedónica influyen de manera significante en la intención de uso. Respecto al uso real, el hábito es la variable más significativa por encima de la intención de uso. Se puede observar que las variables significativas son aquellos valores cuya $\mathrm{P}$ es menor a 0.10 .
Es importante mencionar que se analizó el modelo eliminando la variable de habito y solo se pudo explicar el $9 \%$ del uso real por lo que se concluye que el modelo estructural por mínimos cuadrados tiene más poder predictivo al incluir esta variable.

TABLA IV

ESTADÍSTICAS DE MULTICOLINEALIDAD (VIF)

\begin{tabular}{|l|l|l|l|}
\hline Var & ER & IU & UR \\
\hline CF & & 2.349 & 1.801 \\
\hline CZ & 1.602 & 2.258 & \\
\hline EE & 1.602 & 2.704 & \\
\hline ER & & 2.091 & \\
\hline HB & & 2.087 & 1.97 \\
\hline IU & & & 2.305 \\
\hline IS & & 1.211 & \\
\hline MH & & 2.288 & \\
\hline UR & & & \\
\hline VP & & 1.782 & \\
\hline
\end{tabular}

TABLA V

PRUEBA DE SIGNIFICANCIA

\begin{tabular}{|l|l|l|l|}
\hline Trayectoria & $\begin{array}{l}\text { Valor } \\
\mathbf{T}\end{array}$ & $\begin{array}{l}\text { Valor } \\
\mathbf{P}\end{array}$ & Significancia \\
\hline CF -> IU & 1.422 & 0.155 & No Significativa \\
\hline CF -> UR & 0.66 & 0.509 & No Significativa \\
\hline CZ -> ER & 1.492 & 0.136 & No Significativa \\
\hline CZ -> IU & 5.118 & 0 & Significativa \\
\hline CZ -> UR & 0.594 & 0.552 & No Significativa \\
\hline EE -> ER & 3.259 & 0.001 & Significativa \\
\hline EE -> IU & 0.187 & 0.852 & No Significativa \\
\hline EE -> UR & 0.099 & 0.921 & No Significativa \\
\hline ER -> IU & 1.687 & 0.092 & Significativa \\
\hline ER -> UR & 0.543 & 0.587 & No Significativa \\
\hline HB -> IU & 1.871 & 0.061 & Significativa \\
\hline HB -> UR & 5.317 & 0 & Significativa \\
\hline IU -> UR & 0.612 & 0.541 & No Significativa \\
\hline IS -> IU & 0.186 & 0.852 & No Significativa \\
\hline IS -> UR & 0.097 & 0.923 & No Significativa \\
\hline MH -> IU & 2.048 & 0.041 & Significativa \\
\hline MH -> UR & 0.626 & 0.532 & No Significativa \\
\hline VP -> IU & 1.228 & 0.22 & No Significativa \\
\hline VP -> UR & 0.412 & 0.68 & No Significativa \\
\hline
\end{tabular}




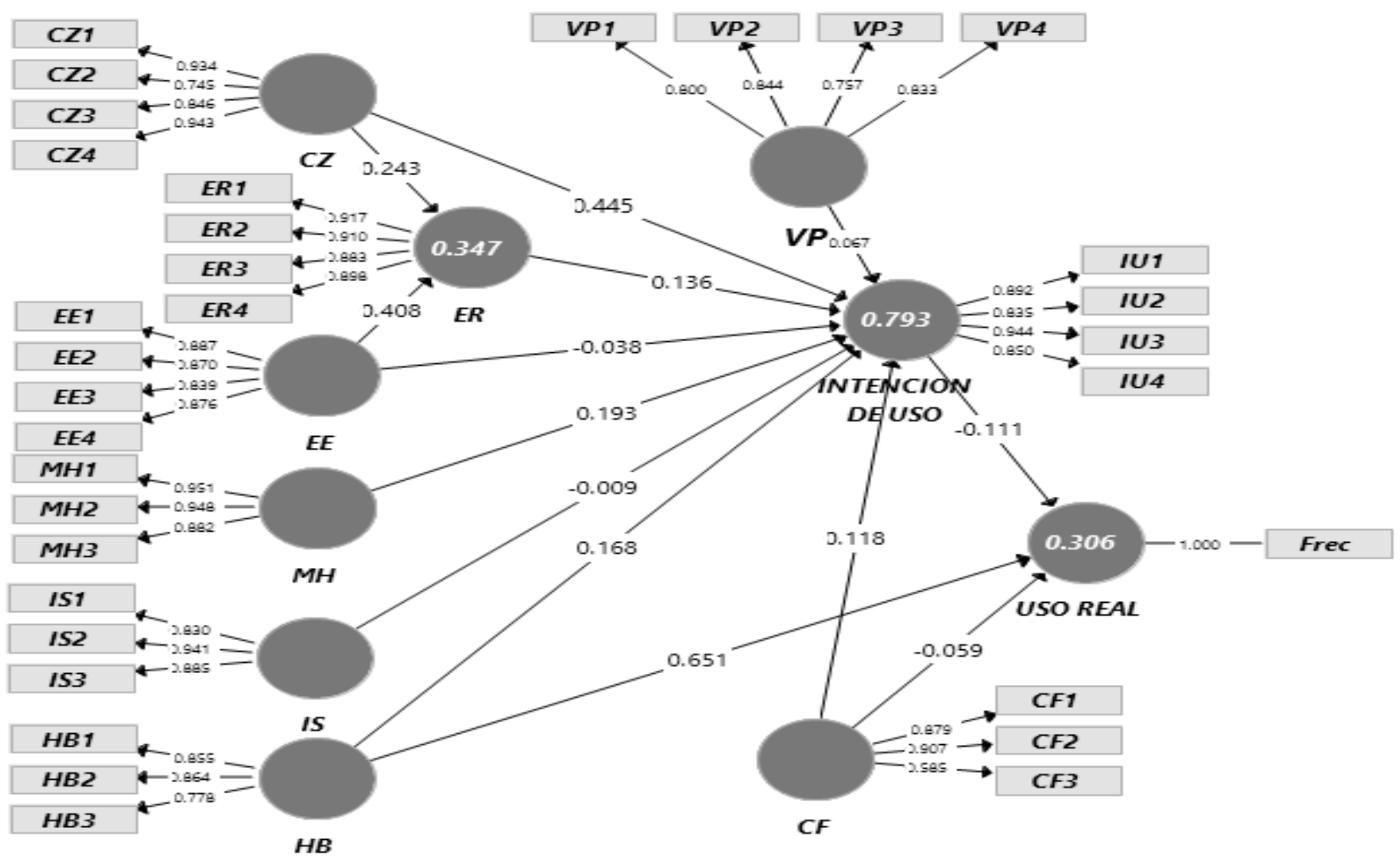

Fig. 2. Resultados del modelo estructural.

\section{DISCUSIÓN}

No se han realizado hasta el momento investigaciones previas de aceptación de criptomonedas en México, para llenar este vacío este estudio utiliza la base teórica del modelo UTAUT2 integrándolo con la confianza, que ha demostrado ser uno de los principales factores en la aceptación de criptomonedas [12]-[14], además el modelo se analiza con la técnica de segunda generación PLS-SEM que posibilita resultados favorables al trabajar con datos no normales, muestras pequeñas y modelos complejos, además esta técnica permite explicar las relaciones causales con un entendimiento más profundo [18].

De acuerdo con los resultados estadísticos presentados anteriormente el modelo que se analiza en el presente estudio alcanza niveles aceptables en cuanto a su nivel predictivo en todos los factores endógenos: la expectativa de rendimiento $34.7 \%$, la intención de uso $79.3 \%$ y el uso real $30.6 \%$. La especificación del modelo también cumple con los criterios mínimos relacionados con la confiabilidad y validez. [44]. La confianza por si sola logra explicar el $44.5 \%$ de la intención de uso por lo que se justifica que este concepto se añadiera al modelo conceptual.

Los resultados revelan que la expectativa de rendimiento tiene un efecto positivo en la intención de usar Bitcoin (H1) y es consistente con estudios previos sobre adopción de criptomonedas [11], [47], en la medida en que el Bitcoin proporciona beneficios que permiten cumplir las tareas del usuario. La expectativa de esfuerzo tiene una influencia positiva en la expectativa de rendimiento por lo que se confirma la hipótesis $\mathrm{H} 2 \mathrm{~b}$, pero no es significativa al explicar la intención de comportamiento para adoptar Bitcoin, resultado consistente con análisis previos [29], [47]. Esto sugiere que un menor esfuerzo en el uso de Bitcoin puede generar mayores expectativas de obtener beneficios, pero no necesariamente implica la adopción de Bitcoin. La influencia social es un factor que ha sido validado significativamente con efectos positivos en estudios de adopción de banca móvil [7], [29], sin embargo en este estudio tiene un efecto negativo sobre la intención de uso (H3) y es consistente con otros estudios sobre adopción de criptomonedas [11], [47]. Este resultado sugiere que la opinión y las recomendaciones de otros no causan efecto en la intención de usar Bitcoin.

A diferencia de otros estudios sobre la adopción de las criptomonedas [11], [12], [47], en el presente estudio no se sustenta la significancia de las condiciones de facilitación (H4a y H4b) al explicar la intención de uso y el uso real de las criptomonedas, esto quiere decir que los usuarios de Bitcoin en México tienen complicaciones técnicas al usar Bitcoin, esto puede deberse a la poca oferta de servicios relacionados con Bitcoin en el país.

Como era de esperarse la motivación hedonista es significativa y tiene un efecto positivo sobre la intención de usar Bitcoin (H5), este resultado es consistente con la referencia [12] que muestra un efecto de alto impacto de la motivación hedónica sobre la intención de uso. Significa que los usuarios de Bitcoin en México experimentan ciertos grados de diversión cuando usan o invierten mediante Bitcoin.

El valor del precio tiene influencia positiva sobre la intención de uso pero este efecto no es significativo por lo que no se 
sustenta la hipótesis H6, resultado en línea con la referencia [12], [29]. El hábito resulta ser el factor más importante sobre el uso real explicando el $65.1 \%$ de la varianza y tiene un efecto positivo moderado sobre la intención de uso. Este constructo no es considerado en estudios sobre nuevas tecnologías [7], [11], [12], [23], sin embargo en el presente estudio si es analizado ya que se considera que los más de 10 años que tiene vigente Bitcoin los usuarios pudieron haber adquirido la experiencia suficiente sobre esta tecnología.

Respecto a los análisis de los coeficientes de trayectoria se encuentra que el factor más significante para predecir la intención de uso es la confianza con un valor de 0.445 (H8a), a su vez tiene un efecto positivo sobre la expectativa de rendimiento (H8b). Esto implica el importante rol de la confianza no solo para motivar a los usuarios de Bitcoin, sino también para crear una percepción positiva sobre la seguridad de esta tecnología, tal como se señala en la referencia [21] en donde se demuestra que el sistema de pagos de Bitcoin en cuanto a su estructura técnica es seguro y confiable.

La intención de usar Bitcoin tiene una connotación negativa sobre el uso real (H9), esto quiere decir que el uso real de Bitcoin por parte de los usuarios se explica más bien por el hábito a través de la confianza. En este sentido se recomienda hacer estudios con muestras más amplias que reflejen mayor amplitud sobre este constructo.

\section{A. Contribuciones teóricas}

Con el creciente impacto que han tenido las criptomonedas y en particular Bitcoin sobre la economía, como su cotización en bolsa, y en específico al ofrecer nuevos productos financieros a través de ellas (FinTech y OIM) es necesario comprender que factores contribuyen a predecir la intención de uso y adopción de Bitcoin por lo que el presente estudio representa una aportación sustancial al área de las criptomonedas. Este estudio contribuye al conocimiento en el contexto mexicano ya que se enfoca en una tecnología poco estudiada que requiere mayor comprensión. Al utilizar una base teórica adecuada en el contexto del usuario como lo es UTAUT2 se amplía la aplicabilidad del modelo al examinarlo en nuevas áreas del conocimiento, sobre todo al analizarlo con métodos de análisis estadísticos avanzados (PLS-SEM). Otra de las aportaciones es la inclusión de un nuevo concepto (confianza) en el modelo UTAUT2 que demuestra una contribución significativa a la teoría. Una contribución teórica relevante es mostrar la existencia de una moneda descentralizada, cuya confianza no reside en una autoridad monetaria, como el dinero Fiat o el plástico.

\section{B. Contribuciones prácticas}

Los resultados estadísticos del presente estudio respaldan el papel crucial de la motivación hedonista, la confianza, la expectativa de rendimiento y el hábito, por lo que todos los aspectos relacionados con estos factores deben de ser el foco de atención de todas aquellas empresas que ofrecen servicios a través de Bitcoin. En la medida que las empresas relacionadas con el Bitcoin destaquen los beneficios que tiene el uso de esta tecnología, como transferencias rápidas y a costos menores, así como destacar que el acceso a esta tecnología no tiene costo, se crearan condiciones que faciliten a los usuarios el acceso a los servicios de Bitcoin en interfaces sencillas.

Se debe tener en consideración la importancia de la confianza sobre la intención de uso, por lo que las empresas relacionadas con Bitcoin deben asegurarse que los usuarios tienen el conocimiento de que a través de esta tecnología pueden realizar transferencias seguras y con altos niveles de confiabilidad. Para ello pueden crear contenido confiables, validados e inteligentes en redes sociales que no solo enseñen como usar Bitcoin sino también métodos para invertir a través de esta tecnología, a la vez estos contenidos pueden contribuir a la motivación hedonista ya que es un factor significante en la intención de uso.

\section{Limitaciones y futuras investigaciones}

El presente estudio tiene algunas limitaciones que propician el estímulo de seguir investigando este tema. Siendo la confianza uno de los factores más importantes en cuanto a la adopción de Bitcoin, una de las principales limitaciones es que el estudio no contempla la confianza multidimensional como se recomienda en [36]. A su vez tampoco se considera el factor de riesgo percibido como un elemento con influencia negativa en la intención de uso como se recomienda en [48] y que debiera ser incluido en todos los análisis relacionados con la confianza como un factor complementario.

La posibilidad de aplicar cuestionarios a través de la red social de Facebook permite tener un mayor alcance en cuanto a la diversidad de los encuestados ya que permite focalizar regiones o países a través de los grupos de criptomonedas, aunque la tasa de respuesta por este medio es muy baja se necesita de más tiempo para hacerla llegar al mayor número de usuarios posibles, esta se considera la principal limitante de este estudio ya que si bien es cierto que alcanzo el número mínimo de respuestas para este tipo de análisis [44], un mayor número hubiese robustecido los resultados.

Dado que este estudio es de corte trasversal, un estudio longitudinal podría proporcionar un mayor alcance en cuanto a la evolución de los factores en el tiempo.

\section{CONCLUSIONES}

Las criptomonedas están recibiendo cada vez más atención, principalmente El Bitcoin el tanto como medio de cambio como de inversión, lo que representa para esta tecnología grandes retos. Tal vez por estar presente el Bitcoin apenas desde 2008, su aceptación y uso aún no han sido evaluados exhaustivamente. A esto hay que añadir que no existen estudios que analicen la adopción de las criptomonedas o el Bitcoin en México. Para llenar esta brecha y cumplir con os objetivos de la presente investigación, se formuló y probó un modelo que integra la teoría UTAUT2 con la confianza. Los resultados estadísticos indican la consistencia, la validez y un alto poder predictivo al explicar un $79,3 \%$ de la varianza de intención de uso y un $30.6 \%$ del uso real de Bitcoin. La expectativa de rendimiento, el hábito, la motivación hedonista y la confianza resultaron ser los factores más significativos para explicar su aceptación como moneda descentralizada. 


\section{AGRADECIMIENTOS}

Los autores reconocemos el apoyo del Centro en Investigación en Alimentación y Desarrollo (AC) en Hermosillo, Sonora, al igual que al Consejo Nacional de Ciencia y Tecnología, para poder realizar esta investigación.

\section{REFERENCIAS}

[1] Y. C. Yeong, K. S. Kalid, and S. K. Sugathan, "Cryptocurrency acceptance: A case of Malaysia," Int. J. Eng. Adv. Technol., vol. 8, no. 5, pp. 28-38, 2019.

[2] S. Nakamoto, "Bitcoin: A peer-to-peer electronic cash system," http://bitcoin.org/bitcoin.pdf," 2008.

[3] CoinMarketCap, "Cryptocurrency Market Capitalizations CoinMarketCap," $2020 . \quad$ [Online]. Available: https://coinmarketcap.com/. [Accessed: 25-May-2020].

[4] C. R. López Zambrano and M. Camberos Castro, "El Bitcoin y las Criptomonedas: ¿Impacto En La Economía?," CAACSX. Memorias del Coloq. Nac. Investig. en las Ciencias Económicas y Adm., vol. 3, no. 3, pp. 409-433, 2019

[5] CoinDance, "Coin Dance | Bitcoin Statistics," 2020. [Online]. Available: https://coin.dance/stats. [Accessed: 12-May-2020].

[6] M. Rauchs, A. Blandin, K. Klein, G. C. Pieters, M. Recanatini, and B. Z. Zhang, "2nd Global Cryptoasset Benchmarking Study," SSRN Electron. J., Mar. 2018.

[7] A. A. Alalwan, Y. K. Dwivedi, and N. P. Rana, "Factors influencing adoption of mobile banking by Jordanian bank customers: Extending UTAUT2 with trust," Int. J. Inf. Manage., vol. 37, no. 3, pp. 99-110, Mar. 2017.

[8] P. Esmaeilzadeh, H. Subramanian, and K. Cousins, "Individuals' cryptocurrency adoption: A proposed moderated-mediation model," 25th Am. Conf. Inf. Syst. AMCIS 2019, no. Saito 2015, pp. 1-10, 2019.

[9] D. Folkinshteyn and M. Lennon, "Braving Bitcoin: A technology acceptance model (TAM) analysis," J. Inf. Technol. Case Appl. Res., vol. 18, no. 4, pp. 220-249, 2016.

[10] A. Walton and K. Johnston, "Exploring perceptions of bitcoin adoption: The South African vir-tual community perspective," Interdiscip. J. Information, Knowledge, Manag., vol. 13, pp. 165-182, 2018.

[11] M. Arias-Oliva, J. Pelegrín-Borondo, and G. Matías-Clavero, "Variables influencing cryptocurrency use: A technology acceptance model in Spain," Front. Psychol., vol. 10, no. MAR, pp. 1-13, 2019.

[12] N. Mahomed, "Understanding consumer adoption of cryptocurrencies," 2017.

[13] C. Roos, "The motivation and factors driving crypto-currency adoption in SMEs," 2015.

[14] F. Shahzad, G. Xiu, J. Wang, and M. Shahbaz, "An empirical investigation on the adoption of cryptocurrencies among the people of mainland China," Technol. Soc., Oct. 2018.

[15] R. Novendra and F. E. Gunawan, "Analysis Of Technology Acceptance And Customer Trust In Bitcoin In Indonesia Using UTAUT Framework," KSII Trans. Internet Inf. Syst., no. January, 2017.

[16] A. T. F. Lou and E. Y. Li, "Integrating innovation diffusion theory and the technology acceptance model: The adoption of blockchain technology from business managers' perspective," Proc. Int. Conf. Electron. Bus., vol. 2017-Decem, pp. 299-302, 2017.

[17] K. Owusu Kwateng, K. A. Osei Atiemo, and C. Appiah, "Acceptance and use of mobile banking: an application of UTAUT2," J. Enterp. Inf. Manag., vol. 32, no. 1, pp. 118-151, 2019.

[18] M. Hair, J. F., Hult, G. T. M., Ringle, C. M., \& Sarstedt, “A Primer on Partial Least Squares Structural Equation Modeling (PLS-SEM). Thousand Oaks," Sage, p. 165, 2013.

[19] B. Inoue, "Performance of Bitcoin Protocol Variants," 2016.

[20] G. Zaera, "Bitcoin: Bases , comportamiento como," Universdade Da Coruña, 2014.

[21] V. Sadhya, H. Sadhya, R. Hirschheim, and E. Watson, "Exploring technology trust in Bitcoin: The blockchain exemplar," 26th Eur. Conf. Inf. Syst. Beyond Digit. - Facet. Socio-Technical Chang. ECIS 2018, pp. $1-16,2018$

[22] V. Venkatesh, M. G. Morris, G. B. Davis, and F. D. Davis, "User acceptance of information technology: Toward a unified view," MIS $Q$. Manag. Inf. Syst., vol. 27, no. 3, pp. 425-478, 2003.
[23] T. Oliveira, M. Faria, M. A. Thomas, and A. Popovič, "Extending the understanding of mobile banking adoption: When UTAUT meets TTF and ITM," Int. J. Inf. Manage., vol. 34, no. 5, pp. 689-703, 2014.

[24] T. Zhou, Y. Lu, and B. Wang, "Integrating TTF and UTAUT to explain mobile banking user adoption," Comput. Human Behav., vol. 26, no. 4, pp. 760-767, Mar. 2010.

[25] I. K. Mensah, L. Chuanyong, and G. Zeng, "Factors determining the continued intention to use mobile money transfer services (MMTS) among university students in Ghana," Int. J. Mob. Hum. Comput. Interact., vol. 12, no. 1, pp. 1-21, 2020.

[26] H. Gunawan, B. L. Sinaga, and S. P. WP, "Assessment of the Readiness of Micro, Small and Medium Enterprises in Using E-Money Using the Unified Theory of Acceptance and Use of Technology (UTAUT) Method," Procedia Comput. Sci., vol. 161, pp. 316-323, 2019.

[27] T. Zhou, Y. Lu, and B. Wang, "Integrating TTF and UTAUT to explain mobile banking user adoption," Comput. Human Behav., vol. 26, no. 4, pp. 760-767, Mar. 2010.

[28] V. Venkatesh, J. Y. L. Thong, and X. Xu, "Consumer acceptance and use of information technology: Extending the unified theory of acceptance and use of technology," MIS Q., vol. 36, no. 1, pp. $157-$ $178,2012$.

[29] T. Oliveira, M. Thomas, G. Baptista, and F. Campos, "Mobile payment: Understanding the determinants of customer adoption and intention to recommend the technology," Comput. Human Behav., vol. 61, no. 2016, pp. 404-414, 2016.

[30] A. A. Alalwan, Y. K. Dwivedi, and N. P. Rana, "Factors influencing adoption of mobile banking by Jordanian bank customers: Extending UTAUT2 with trust," Int. J. Inf. Manage., vol. 37, no. 3, pp. 99-110, 2017.

[31] M. A. Mahfuz, L. Khanam, and H. Wang, "The influence of culture on m-banking technology adoption: An integrative approaches of UTAUT2 and ITM," PICMET 2016 - Portl. Int. Conf. Manag. Eng. Technol. Technol. Manag. Soc. Innov. Proc., pp. 824-835, 2017.

[32] N. Mahomed, "Understanding consumer adoption of cryptocurrencies," 2017.

[33] N. Rodenrijs and J. Wokke, "Will social media make or break the acceptance in new technology? A quantitative study of consumer acceptance in cryptocurrency," 2018.

[34] F. Shahzad, G. Y. Xiu, J. Wang, and M. Shahbaz, "An empirical investigation on the adoption of cryptocurrencies among the people of mainland China," Technol. Soc., vol. 55, no. May, pp. 33-40, 2018.

[35] A. Walton and K. Johnston, "Exploring perceptions of bitcoin a doption : the south african virtual community perspective," vol. 13, pp. 165-182, 2018.

[36] D. Gefen, E. Karahanna, and W. Straub, Detmar, "Trust and TAM in Online Shopping: An Integrated Model," MIS Quart., vol. 27, no. 1, pp. 51-90, 2003.

[37] V. Venkatesh, M. G. Morris, G. B. Davis, and F. D. Davis, "User acceptance of information technology: Toward a unified view," MIS $Q$. Manag. Inf. Syst., vol. 27, no. 3, pp. 425-478, Mar. 2003.

[38] F. D. Davis, R. P. Bagozzi, and P. R. Warshaw, "User Acceptance of Computer Technology: A Comparison of Two Theoretical Models," Manage. Sci., vol. 35, no. 8, pp. 982-1003, 1989.

[39] S. Afshan and A. Sharif, "Acceptance of mobile banking framework in Pakistan," Telemat. Informatics, vol. 33, no. 2, pp. 370-387, 2016.

[40] T. Zhou, Y. Lu, and B. Wang, "Integrating TTF and UTAUT to explain mobile banking user adoption," Comput. Human Behav., vol. 26, no. 4, pp. 760-767, 2010.

[41] V. Venkatesh, J. Y. L. Thong, and X. Xu, "Venkatesh_Thong_Xu_MISQ_forthcoming (Gender Age Experience)," MIS Q., vol. 36, no. 1, pp. 157-178, 2012.

[42] T. Zhou, "Examining mobile banking user adoption from the perspectives of trust and flow experience," Inf. Technol. Manag., vol. 13, no. 1, pp. 27-37, 2012.

[43] I. Ajzen, "The Theory of Planned Behavior," Organ. Behav. Hum. Decis. Process., vol. 50, no. 1, pp. 179-211, 1991.

[44] J. F. Hair, M. Sarstedt, C. M. Ringle, and J. A. Mena, "An assessment of the use of partial least squares structural equation modeling in marketing research," J. Acad. Mark. Sci., vol. 40, no. 3, pp. 414-433, 2012.

[45] O. Leyva Cordero and J. T. Olague, Modelo de Ecuaciones Estructurales por el método de mínimos cuadrados parciales (PLS), no. April 2015. 2014.

[46] J. F. Hair, M. Sarstedt, L. Hopkins, and V. G. Kuppelwieser, "Partial 
least squares structural equation modeling (PLS-SEM): An emerging tool in business research," Eur. Bus. Rev., vol. 26, no. 2, pp. 106-121, 2014.

[47] M. A. Mahfuz, L. Khanam, and W. Hu, "The influence of culture on m-banking technology adoption: An integrative approaches of UTAUT2 and ITM," in 2016 Portland International Conference on Management of Engineering and Technology (PICMET), 2016, pp. 824-835.

[48] D. Gefen, V. Srinivasan Rao, and N. Tractinsky, "The conceptualization of trust, risk and their electronic commerce: The need for clarifications," Proc. 36th Annu. Hawaii Int. Conf. Syst. Sci. HICSS 2003, vol. 00, no. C, pp. 1-10, 2003.

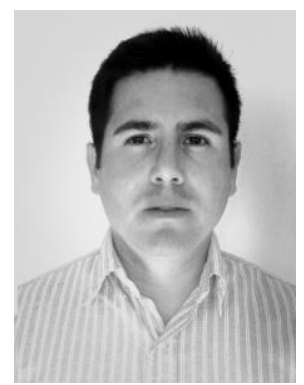

Carlos Roberto López Zambrano. Licenciado en Derecho, 2008, por la Universidad de Sonora y Maestro en Comercio Exterior y Aduanas, 2015, Universidad de Sonora. Estudiante de Doctorado en Desarrollo Regional en el Centro de Investigación en Alimentación y Desarrollo, CIAD, de Hermosillo, Sonora, México. Actualmente docente de tiempo parcial en el programa de Administración y Comercio en la Universidad Tec Milenio Campus Hermosillo, México.

ORCID: https://orcid.org/0000-0002-7880-7387

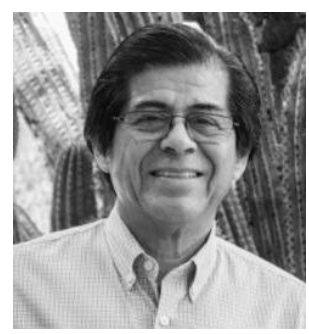

Mario Camberos Castro. Es Licenciado en Economía por la Universidad de Guadalajara. Cuenta con una Maestría en Ciencias Económicas por la Universidad Nacional Autónoma de México. Es Doctor en Ciencias Económicas por la Universidad Autónoma de Baja California. Es profesor investigador emérito del Centro de Investigación en Alimentación y Desarrollo. Actualmente es Investigador Titular del Departamento de Economía, Coordinación de Desarrollo Regional, Hermosillo del Centro de Investigación en Alimentación y Desarrollo, CIAD. Imparte las materias de Macroeconomía y Modelos de Desarrollo en México, Métodos y Técnicas de Medición de la Pobreza, Filosofía de la ciencia, Crecimiento y Desigualdad.

ORCID: https://orcid.org/0000-0002-3271-2980 